\title{
Inflammatory breast cancer in 210 patients: A retrospective study on epidemiological, anatomo-clinical features and therapeutic results
}

\author{
MAROUA MANAI $^{1-4}$, PASCAL FINETTI ${ }^{4}$, NESRINE MEJRI ${ }^{2}$, SALMA ATHIMNI $^{2}$, DANIEL BIRNBAUM ${ }^{4}$, \\ FRANÇOIS BERTUCCI ${ }^{4-6}$, KHALED RAHAL ${ }^{1}$, AMOR GAMOUDI $^{1}$, MAX CHAFFANET $^{4}$, \\ MOHAMED MANAI $^{3 *}$ and HAMOUDA BOUSSEN ${ }^{2,3,7^{*}}$
}

\begin{abstract}
${ }^{1}$ Department of Immuno-Histo-Cytology, Salah Azaiez Institute, Tunis 1006; ${ }^{2}$ Medical Oncology Service, University Hospital Abderrahmane Mami, Ariana 2080; ${ }^{3}$ Laboratory of Biochemistry and Molecular Biology, Department of Biology, Faculty of Sciences, University of Tunis El Manar, Ariana 2092, Tunisia; ${ }^{4}$ Predictive Oncology Team, Marseille Cancer Research Center (INSERM UMR1068; IFR137), Paoli-Calmettes Institute; ${ }^{5}$ UFR of Medicine, Aix Marseille University; ${ }^{6}$ Department of Medical Oncology, Marseille Cancer Research Center (INSERM UMR1068; IFR137), Paoli-Calmettes Institute, Aix Marseille University, 13009 Marseille; ${ }^{7}$ Oncology Radiotherapy Association for the Mediterranean, 75014 Paris, France
\end{abstract}

Received March 12, 2018; Accepted October 1, 2018

DOI: $10.3892 / \mathrm{mco} .2018 .1773$

\begin{abstract}
To report epidemiological and anatomo-clinical features within a retrospective series of inflammatory breast cancer and to evaluate prognostic factors. This retrospective study included 210 Tunisian patients presenting a clinically diagnosed IBC, treated at the Institute Salah Azaiez (ISA) of Tunis, Tunisia, from 2008 to 2013. We collected data on epidemiology, anatomo-clinical and biological features and histologic response to neoadjuvant therapy. Overall and disease-free survivals were calculated by Kaplan-Meier method and compared by log-rank tests and Cox's models were used to identify prognostic factors impacting survival. The 210 IBC patients had a median age of 42 years (24-62) and 15\% of them were aged less than 35 years. Mean age at menarche was 13 years and $45 \%$ had their 1st childbirth before 23 years. On histology, grades III represented $42 \%$ of cases, hormone receptors were negative in 59\%, HER2 over-expressed in $32,25 \%$ of our IBC cases had a triple negative profile and $\mathrm{Ki}-67$ was $>20 \%$ in $53 \%$ of cases. High pathological grade III was significantly correlated to TN subtype (58\%) (Fisher's exact test, $\left.\mathrm{P}=7.5 \times 10^{-3}\right)$. Further, high Ki-67 expression $(>20 \%)$ was evident in the TN subtype (84\%) (Fisher's exact

Correspondence to: Dr Maroua Manai, Department of Immuno-Histo-Cytology, Salah Azaiez Institute, Place Bab Saadoun, Tunis 1006, Tunisia

E-mail: maroua.m@hotmail.com

${ }^{*}$ Contributed equally
\end{abstract}

Key words: inflammatory breast cancer, epidemiology, histology, immunohistochemistry, chemotherapy response, prognosis, survival test, $\mathrm{P}=3.7 \times 10^{-4}$ ). After neoadjuvant therapy (and trastuzumab in 88 and $69 \%$ of HER2+ patients, respectively), we observed $49 \%$ of objective clinical responses and $35 \%$ of pathological complete response (pCR) and $>3$ axillary lymph nodes were invaded on a resected tumor in $55 \%$ of cases. Overall survival (OS) was associated with age at menarche (Wald-test, $\mathrm{P}=2.2 \times 10^{-2}$ ) and metastases at diagnosis (Wald-test, $\mathrm{P}=2.4 \times 10^{-2}$ ). Reaching a pCR was correlated with a better metastasis-free survival (MFS), (Fisher's exact test, $\mathrm{P}=3.6 \times 10^{-2}$ ).

\section{Introduction}

Inflammatory Breast Cancer (IBC) is a particular and aggressive variety of breast cancers (BC), with a high metastatic potential. IBC is a rare form of breast cancer representing $2 \%$ of $\mathrm{BC}$ in Europe and USA lower than that (5-10\%) in North Africa and Tunisia (1-3). Diagnosis is based on strict clinical criteria's and this entity is classified as a T4d tumor according to the TNM-UICC (4). IBC is a rapidly evolutive disease, with clinical signs, occurring in less than six months presented by edema, erythema, breast enlargement and 'peau d'orange' (3). Despite advances obtained through a multidisciplinary approach by, combining neoadjuvant chemo and/or targeted therapy-mastectomy and loco-regional radiotherapy, prognosis remains poor, with 5-years overall and disease-free survival $<50 \%$ compared to $\sim 70 \%$ for 'neglected' T4b non-inflammatory breast cancers (non-IBCs) (5).

This retrospective study aimed to report the epidemiologic, anatomoclinical and therapeutic features and to evaluate the role of prognostic factors within an extensive series of 210 Tunisian patients with IBC treated from 2008 to 2013.

\section{Materials and methods}

Patients and samples. We collected 210 cases of IBC, treated between 2008 and 2013 at Salah Azaiez Institute (ISA) in 
Tunis, Tunisia. Main inclusion criteria were female sex, with clinically defined and pathologically confirmed IBC (T4d), written informed consent, available formaldehyde-fixed and paraffin-embedded pre-therapeutic diagnostic tumor sample, and comprehensive clinic-pathological data. These features included: Age at diagnosis, TNM stage, pathological plus immunohistochemical data such as histologic type and grade, pathological tumor size, pathological complete response, axillary lymph node status of Estrogen (ER)/Progesterone (PR) receptors, HER2 and Ki-67 status, treatment and clinical outcome. The molecular subtypes of tumors were defined according to immunohistochemistry (IHC). The study was approved by our institutional ethics committee at Salah Azaiez Institute.

Immunohistochemistry analysis. IHC staining of the paraffin blocks was routinely carried out for an immune-peroxidase assay for ER, PR and HER2 (Novocastra, Laboratories, Ltd., Newcastle upon Tyne, UK). The $4 \mu \mathrm{m}$ thick tissue sections were cleared in xylene, rehydrated in ethanol and rinsed in distilled water. The slides were then incubated with specific primary antibodies for $30 \mathrm{~min}$ at room temperature and the reaction revealed through incubation with hydrogen-peroxide and a chromogen agent diaminobenzidine for $10 \mathrm{~min}$ and counterstained with hematoxylin. The slides were then dehydrated and mounted for local pathologists to evaluate the IHC staining of sections from paraffin. Only the nuclear reactivity was taken into account for steroid hormone receptors (HR) to define the presence or absence of ER, PR in $<5 \%$ of the neoplastic cells. Tumor scores of 0 or $1+$ were considered to be HER2 negative (HER2-) whereas those scoring 3+ with strong complete membrane staining were considered to be HER2 positive (HER2+). The tumors with an IHC score of HER 2 2+ were assayed with colorimetric in situ hybridization (CISH) using (HER2 probe kit; ZytoDot SPEC Molecular Diagnostics, Bremerhaven, Germany). CISH was scored on a standard quantitative scale in which less than six copies of the HER2 gene are classified as negative. The molecular subtypes of tumors were based on ER, PR and HER2 IHC statutes and included luminal A or B (ER+/PR+/HER2-), luminal B HER2+ (ER+/PR+/HER2+), HER2+ (ER-/PR-/HER2+), and triple negative (ER-/PR-/HER2-). The immunohistochemistry detection of Ki-67 (1/200 Novocastra, incubation $1 \mathrm{~h}$ at pH9) was carried out as previously described (6).

The revelation was performed using the Dako Flex system (Dako, Agilent Technologies, Inc., Santa Clara, CA, USA) in DAB. Sections counterstained with hematoxylin were independently evaluated by light microscopy by two experienced breast pathologists. Immunoreactivities were scored mainly by measuring the percentage of positive tumor cells, from $0 \%$ for the undetectable level to $100 \%$ for total homogeneous staining.

Statistical analysis. Data were summarized by numbers and percentages for categorical variables, and median and range for continuous variables. Correlations between tumor groups and clinicopathological features were analyzed using the two-sample t-test or the Fisher's exact test when appropriate. Follow-up was calculated from the date of diagnosis to the date of last news for event-free patients. Metastasis-free survival (MFS) was calculated from the date of diagnosis until the date of first distant relapse. Overall survival (OS) was calculated from the date of diagnosis until the date of breast cancer-related death. Univariate prognostic analyses for OS and MFS were done using Cox regression analysis (Wald test). All statistical tests were two-sided at the 5\% level of significance. Analyses were performed by the survival package (version 2.30) in the R software (version 2.9.1; www.cran.r-project.org/).

\section{Results}

IBC epidemiological and clinicopathological features. We analyzed the clinicopathological features about our series of 210 IBC patients with known receptor status treated from 2008 and 2013 at the Salah Azaiez Institute of Tunis are listed in Table I. Their mean age was relatively young at 42 years (24-62) and $15 \%$ were younger than 35 years. Mean age at menarche was 13 years. Mean age at first childbirth was at 23 years in $45 \%$ of cases and $54 \%$ of patients never received oral contraception. On histology, $42 \%$ of tumors were grade SBR III, 59\% were HR-negative and 32\% overexpressed HER2. IHC subtypes were HR-negative/HER2-positive in 27\%; HR-positive/HER2-negative in 43\%, HR-positive/HER2-positive in $5 \%$ and $\mathrm{TN}$ in $25 \%$ (Fig. 1) while Ki-67 rate higher than $20 \%$ was in $53 \%$ of cases. Neoadjuvant treatment (NAT) consisted in the sequential protocol (anthracylines-taxanes in 88\%) and 69\% of HER2+ cases received trastuzumab. We observed after NAT, $49 \%$ of objective clinical responses and $35 \%$ of pCR, and most patients $(55 \%)$ had more than three axillary lymph nodes invaded.

Correlation of clinical outcomes. In Table I, the four subgroups did not differ significantly in age, mean age at menarche, mean age at first birth before 23 years, or contraception status. There were no differences by subtype in clinical node status, lymphatic involvement. However, high pathological grade III was significantly correlated to TN subtype (58\%) (Fisher's exact test, $\left.\mathrm{P}=7.5 \times 10^{-3}\right)$. Further, high Ki-67 expression $(>20 \%)$ was evident in the TN subtype (84\%) (Fisher's exact test, $\left.\mathrm{P}=3.7 \times 10^{-4}\right)$. We found no differences in the distribution of neoadjuvant regimens, clinical response or $\mathrm{pCR}$ among the four groups. In term of pCR rate, we observed that the obtention of pCR was correlated with a better MFS (Fisher's exact test, $\mathrm{P}=3.6 \times 10^{-2}$ ). We didn't find any anatomoclinical parameter correlated with pCR (Table II). On univariate analysis concerning 154 patients, poor OS was correlated with younger age at menarche [Wald-test, $\mathrm{P}=2.2 \times 10^{-2}, \mathrm{HR}=1.97$ (1.10-3.55)] and initial metastases [(Wald-test, $\left.\mathrm{P}=2.4 \times 10^{-2}\right)$, $H R=17.2(2.73-108)]$. No correlation was found with the other clinicopathological factors or with the four subgroups (Table III). For MFS, univariate analysis concerned 160 non-metastatic (M0) patients at diagnosis and a better MFS was observed in the presence of an objective clinical response to NAT [Wald-test, $\mathrm{P}=2.3 \times 10^{-2}, \mathrm{HR}=0.28(0.10-0.84)$ ] and tended to be associated with the presence of pCR [Wald-test, $\left.\mathrm{P}=5.4 \times 10^{-2}, \mathrm{HR}=0.23(0.05-1.03)\right]$. None correlation, good or poor, was found between a poor MFS and the other clinicopathological factors or the four subtypes (Table IV).

\section{Discussion}

We have reported here clinical and biological data from a large cohort of 210 Tunisian patients with IBC treated 
Table I. Epidemiological and clinicopathological characteristics of patients (2008-2013).

\begin{tabular}{|c|c|c|c|c|c|c|}
\hline \multirow[b]{2}{*}{ Characteristics } & \multirow[b]{2}{*}{$\mathrm{N}=210$} & \multicolumn{4}{|c|}{ HR/HER2 status } & \multirow[b]{2}{*}{ P-value } \\
\hline & & HR-/HER2+ & HR+/HER2- & HR+/HER2+ & $\mathrm{TN}$ & \\
\hline Age, years, mean (range) & $42(24-62)$ & $42(27-62)$ & $42(24-61)$ & $41.5(31-51)$ & $42(28-60)$ & 0.64 \\
\hline$<35$ & $32(15 \%)$ & $6(12 \%)$ & $12(14 \%)$ & $2(25 \%)$ & $11(22 \%)$ & 0.36 \\
\hline$\geq 35$ & $175(85 \%)$ & $46(88 \%)$ & $71(86 \%)$ & $6(75 \%)$ & $38(78 \%)$ & \\
\hline First menarche, mean year (range) & $13(9-18)$ & $13(10-18)$ & $13(9-18)$ & $12(11-15)$ & $13(10-16)$ & 0.47 \\
\hline \multicolumn{7}{|l|}{ First child, years } \\
\hline$<23$ & $68(45 \%)$ & $16(40 \%)$ & $30(50 \%)$ & $3(50 \%)$ & $16(43 \%)$ & 0.79 \\
\hline$\geq 23$ & $84(55 \%)$ & $24(60 \%)$ & $30(50 \%)$ & $3(50 \%)$ & $21(57 \%)$ & \\
\hline \multicolumn{7}{|l|}{ Contraception } \\
\hline No & $97(54 \%)$ & $29(63 \%)$ & $43(57 \%)$ & $4(50 \%)$ & $16(39 \%)$ & 0.13 \\
\hline Yes & $82(46 \%)$ & $17(37 \%)$ & $32(43 \%)$ & $4(50 \%)$ & $25(61 \%)$ & \\
\hline \multicolumn{7}{|l|}{ Pathological type } \\
\hline Ductal & $209(100 \%)$ & $52(98 \%)$ & $83(100 \%)$ & $9(100 \%)$ & $49(100 \%)$ & 0.57 \\
\hline Mixed & $1(0 \%)$ & $1(2 \%)$ & $0(0 \%)$ & $0(0 \%)$ & $0(0 \%)$ & \\
\hline \multicolumn{7}{|l|}{ Pathological grade } \\
\hline I-II & $100(58 \%)$ & $24(52 \%)$ & $50(68 \%)$ & $5(100 \%)$ & $18(42 \%)$ & $7.50 \times 10^{-3}$ \\
\hline III & $73(42 \%)$ & $22(48 \%)$ & $24(32 \%)$ & $0(0 \%)$ & $25(58 \%)$ & \\
\hline \multicolumn{7}{|l|}{ HR } \\
\hline Negative & $114(59 \%)$ & $53(100 \%)$ & $12(14 \%)$ & $0(0 \%)$ & $49(100 \%)$ & $<1.0 \times 10^{-6}$ \\
\hline Positive & $80(41 \%)$ & $0(0 \%)$ & $71(86 \%)$ & $9(100 \%)$ & $0(0 \%)$ & \\
\hline \multicolumn{7}{|l|}{ HER 2} \\
\hline Negative & $133(68 \%)$ & $0(0 \%)$ & $83(100 \%)$ & $0(0 \%)$ & $49(100 \%)$ & $<1.0 \times 10^{-6}$ \\
\hline Positive & $64(32 \%)$ & $53(100 \%)$ & $0(0 \%)$ & $9(100 \%)$ & $0(0 \%)$ & \\
\hline \multicolumn{7}{|l|}{$\mathrm{Ki} 67, \%$} \\
\hline$<20$ & $51(47 \%)$ & $15(44 \%)$ & $30(65 \%)$ & $2(67 \%)$ & $4(16 \%)$ & $3.70 \times 10^{-4}$ \\
\hline$\geq 20$ & $57(53 \%)$ & $19(56 \%)$ & $16(35 \%)$ & $1(33 \%)$ & $21(84 \%)$ & \\
\hline \multicolumn{7}{|l|}{ Nodal involvement } \\
\hline$\leq 3$ nodes & $66(45 \%)$ & $22(61 \%)$ & $23(38 \%)$ & $3(50 \%)$ & $14(38 \%)$ & 0.12 \\
\hline$>3$ nodes & $80(55 \%)$ & $14(39 \%)$ & $37(62 \%)$ & $3(50 \%)$ & $23(62 \%)$ & \\
\hline \multicolumn{7}{|l|}{ TNM } \\
\hline N0 & $10(5 \%)$ & $1(2 \%)$ & $3(4 \%)$ & $0(0 \%)$ & $3(6 \%)$ & 0.68 \\
\hline $\mathrm{N} 1-3$ & $198(95 \%)$ & $52(98 \%)$ & $79(96 \%)$ & $9(100 \%)$ & $46(94 \%)$ & \\
\hline \multicolumn{7}{|l|}{ TNM } \\
\hline M0x & $184(88 \%)$ & $45(85 \%)$ & $73(88 \%)$ & $8(89 \%)$ & $46(94 \%)$ & 0.5 \\
\hline M1 & $25(12 \%)$ & $8(15 \%)$ & $10(12 \%)$ & $1(11 \%)$ & $3(6 \%)$ & \\
\hline \multicolumn{7}{|l|}{ Neoadjuvant treatment } \\
\hline No & $203(12 \%)$ & $8(15 \%)$ & $8(11 \%)$ & $1(11 \%)$ & $4(8 \%)$ & 0.6 \\
\hline Yes & $179(88 \%)$ & $45(85 \%)$ & $63(89 \%)$ & $8(89 \%)$ & $45(92 \%)$ & \\
\hline \multicolumn{7}{|l|}{ Trastuzumab (of HER2+ patients) } \\
\hline No & $20(31 \%)$ & $16(30 \%)$ & $0(0 \%)$ & $2(22 \%)$ & $0(0 \%)$ & 1 \\
\hline Yes & $45(69 \%)$ & $37(70 \%)$ & $0(0 \%)$ & $7(78 \%)$ & $0(0 \%)$ & \\
\hline \multicolumn{7}{|l|}{ Clinical response } \\
\hline No & $67(51 \%)$ & $20(57 \%)$ & $28(51 \%)$ & $3(60 \%)$ & $14(48 \%)$ & 0.89 \\
\hline Yes & $64(49 \%)$ & $15(43 \%)$ & $27(49 \%)$ & $2(40 \%)$ & $15(52 \%)$ & \\
\hline \multicolumn{7}{|l|}{ Pathological complete response } \\
\hline No & $66(65 \%)$ & $18(67 \%)$ & $28(60 \%)$ & $4(80 \%)$ & $13(68 \%)$ & 0.79 \\
\hline Yes & $36(35 \%)$ & $9(33 \%)$ & $19(40 \%)$ & $1(20 \%)$ & $6(32 \%)$ & \\
\hline
\end{tabular}


Table I. Continued.

\begin{tabular}{|c|c|c|c|c|c|c|}
\hline \multirow[b]{2}{*}{ Characteristics } & \multirow[b]{2}{*}{$\mathrm{N}=210$} & \multicolumn{4}{|c|}{ HR/HER2 status } & \multirow[b]{2}{*}{ P-value } \\
\hline & & HR-/HER2+ & HR+/HER2- & HR+/HER2+ & $\mathrm{TN}$ & \\
\hline \multicolumn{7}{|c|}{ Molecular subtypes } \\
\hline HR-/HER2+ & $53(27 \%)$ & & & & & \\
\hline HR+/HER2- & $83(43 \%)$ & & & & & \\
\hline HR+/HER2+ & $9(5 \%)$ & & & & & \\
\hline $\mathrm{TN}$ & $49(25 \%)$ & & & & & \\
\hline
\end{tabular}

HR, hormonal receptor; HER2, human epidermal growth factor receptor 2; TNM, tumor-nodes-metastasis; TN, triple negative.
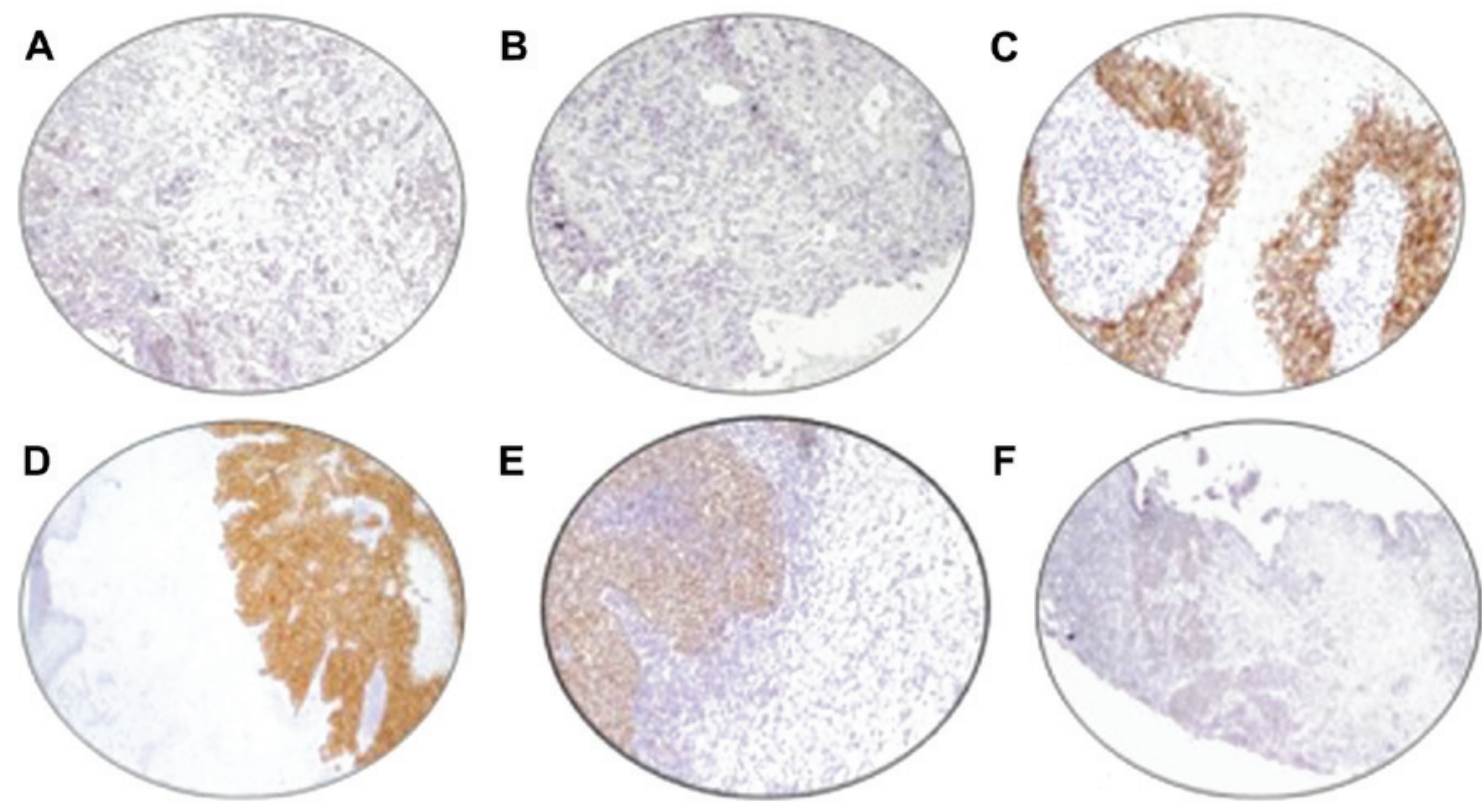

Figure 1. Immunohistochemistry in inflammatory breast cancer. (A and B) Absence of expression of ER and PR in IBC tumor cases. (C) Strong expression of HER2 in IBC cases (Score 3). (D and E) Expression of ER and PR in 100\% of tumors cells in IBC cases. (F) Absence of expression of HER2 in IBC cases (magnification, x200). ER, estrogen receptor; PR, progesterone receptor; IBC, inflammatory breast cancer; HER2, human epidermal growth factor receptor 2.

from 2008 to 2013 and classified as T4d by using stringent diagnosis criteria, treated at the Salah Azaiez Institute of Tunis, Tunisia, a comprehensive center, having a huge clinical expertise in the field of IBC for $>50$ years $(7,8)$. The clinical IBC entity represents $5-7 \%$ of BCs in Tunisia and its frequency was readjusted by applying more strict diagnosis criteria, compared to the higher (30-55\% of BCs) reported in the seventies by Mourali team $(1,8,9)$. In North Africa, Algeria, Morocco and Tunisia constitute a cluster with high IBC incidence compared to occidental countries, where this entity account for $<2 \%$ of $\mathrm{BCs}(7,10,11)$. A correct diagnosis of IBC is crucial and should be based on an amnestic evolution of symptoms in less than 3 months, combined with the presence of local inflammatory and/or regional, i.e., erythema, edema, skin thickness ('peau d'orange'), involving more than $1 / 3$ of breast (3). It is essential to differentiate IBC from the locally advanced non-IBC (LABC) and neglected BC that occur in older patients ( $>70$ years), with evolution within six months or more and a frequent delay in consultation and diagnosis. This confusion between IBC and $\mathrm{LABC}$ remains the reported studies from occidental countries, wherein IBC series, the average age is ten years or more, higher than that observed in Tunisia, over 55 years (12). In the Surveillance, Epidemiology and End Results (SEER) series (12), including a retrospective cohort collected from 1992-2002, the older age reported for patients is probably biased by the absence of documented diagnostic reviews and the inclusion of elderly patients probably presenting a higher enrichment in LABCs than IBC entities (13). The same age difference between occidental and North African series, exceeding ten years for mean age at IBC diagnosis (55 vs. 42 years) is observed in the series from the MD Anderson Cancer Center and those we studied (per the M.D Anderson IBC Registry) (7). Most IBC epidemiologic and risk factors data were previously reported in Tunisian, US and French studies $(7,8,14,15)$. Retrospective data suggest as risk factors, a young age ( $<12$ years old) at 1 st menarche and first birth between 20-23-year-old $(7,8,14,16)$. A frequent association between IBC, pregnancy and a 
Table II. Pathological complete response and clinicopathological features.

\begin{tabular}{|c|c|c|c|}
\hline \multirow[b]{2}{*}{ Characteristics } & \multicolumn{2}{|c|}{$\mathrm{pCR}$} & \multirow[b]{2}{*}{ P-value } \\
\hline & No & Yes & \\
\hline Age, mean year (range) & $42(28-61)$ & $42(24-60)$ & 0.99 \\
\hline Age, $<35$ years $(\%)$ & 17 & 19 & 0.79 \\
\hline First menarche, mean year (range) & $13(9-18)$ & $13(10-18)$ & 0.93 \\
\hline First child, $<23$ years $(\%)$ & 49 & 52 & 1.00 \\
\hline Contraception, no (\%) & 39 & 39 & 1.00 \\
\hline Pathological type, ductal (\%) & 98 & 100 & 1.00 \\
\hline Pathological grade, III (\%) & 41 & 52 & 0.37 \\
\hline HR, negative $(\%)$ & 52 & 66 & 0.29 \\
\hline HER2, postive (\%) & 35 & 31 & 0.67 \\
\hline $\mathrm{Ki67}, \geq 20 \%(\%)$ & 36 & 52 & 0.27 \\
\hline TNM, N1-3 (\%) & 94 & 91 & 0.35 \\
\hline TNM, M0x (\%) & 94 & 92 & 0.70 \\
\hline 5-year MFS, \% ( $\left.\mathrm{IC}_{95}\right)$ & $37(15-91)$ & $48(12-100)$ & $3.6 \times 10^{-2}$ \\
\hline
\end{tabular}

pCR, pathological complete response; HR, hormonal receptor; HER2, human epidermal growth factor receptor 2; N, nodes; M, metastasis; MFS, metastasis free survival.

Table III. Univariate prognostic analysis for overall survival.

\begin{tabular}{|c|c|c|c|}
\hline \multirow[b]{2}{*}{ Characteristics } & \multicolumn{3}{|c|}{ Univariate analysis } \\
\hline & $\mathrm{N}$ & HR $(95 \%$ CI $)$ & P-value \\
\hline Age & 153 & $1.00(0.92-1.10)$ & 0.93 \\
\hline Age, $<35$ vs. $\geq 35$ years & 153 & $1.30(0.15-11.2)$ & 0.81 \\
\hline First menarche & 140 & $1.97(1.10-3.55)$ & $2.2 \times 10^{-2}$ \\
\hline First child, $<23$ vs. $\geq 23$ years & 115 & $1.77(0.29-10.8)$ & 0.54 \\
\hline Contraception, yes vs. no & 134 & $3.59(0.40-32.3)$ & 0.25 \\
\hline Pathological type, ductal vs. other & 154 & 0.00 (0.00-Inf) & 1.00 \\
\hline Pathological grade, III vs. I-II & 132 & $0.65(0.10-4.18)$ & 0.65 \\
\hline HR, postive vs. negative & 147 & $0.37(0.04-3.16)$ & 0.36 \\
\hline HER2, postive vs. negative & 148 & $1.13(0.20-6.25)$ & 0.89 \\
\hline \multicolumn{4}{|l|}{ Molecular subtypes } \\
\hline HR-/HER2+ vs. HR+/HER2- & 147 & $1.42(0.20-10.1)$ & 0.98 \\
\hline HR+/HER2+ vs. HR+/HER2- & & 0.00 (0.00-Inf) & \\
\hline TN vs. HR+/HER2- & & $0.98(0.13-7.14)$ & \\
\hline Ki67, $\geq 20 \%$ vs. $<20 \%$ & 84 & $0.31(0.03-3.53)$ & 0.34 \\
\hline TNM, N1-3 vs. N0 & 153 & $9.6 \mathrm{E}+06(0-\mathrm{Inf})$ & 1.00 \\
\hline TNM, M1 vs. M0x & 154 & $17.2(2.73-108)$ & $2.4 \times 10^{-2}$ \\
\hline Clinical response, yes vs. no & 104 & $0.00(0.00-\operatorname{Inf})$ & 1.00 \\
\hline pCR, yes vs. no & 78 & $1.40(0.09-22.5)$ & 0.81 \\
\hline
\end{tabular}

Concerned 154 patients with an available survival information. HR, hormonal receptor; HER2, human epidermal growth factor receptor 2; TN, triple negative; N, nodes; M, metastasis; pCR, pathological complete response; HR, hazard ratio; CI, confidence interval.

prolonged period of breastfeeding, exceeding 24 months was also reported (17). In our series, mean age at menarche has occurred at 13 year-old, and first childbirth was before 23 years in $45 \%$ of cases.
In our series, we found that clinical IBC aggressiveness is associated, compared to 'classical BC' with a higher frequency of HR-negative, HER 2+ and TN subtypes (59, 32 and 25\% of cases, respectively). Such data are in agreement with those reported 
Table IV. Univariate prognostic analyses for metastasis-free survival.

Univariate analysis

\begin{tabular}{lccc} 
& $\mathrm{N}$ & HR $(95 \%$ CI $)$ & P-value \\
\cline { 2 - 4 } Characteristics & 160 & $0.98(0.94-1.02)$ & $0.85(0.37-1.97)$ \\
Age & 160 & $1.07(0.89-1.28)$ & 0.32 \\
Age, $<35$ vs. $\geq 35$ years & 145 & $1.81(0.84-3.94)$ & $1.07(0.50-2.29)$ \\
First menarche & 120 & $0.00(0.00-$ Inf $)$ & 0.49 \\
First child, <23 vs. $\geq 23$ years & 142 & $1.27(0.62-2.57)$ \\
Contraception, yes vs. no & 161 & $1.11(0.54-2.30)$ \\
Pathological type, ductal vs. other & 143 & $0.75(0.33-1.67)$ \\
Pathological grade, III vs. I-II & 154 & & 0.13 \\
HR, postive vs. negative & 155 & $0.83(0.33-2.11)$ \\
HER2, postive vs. negative & & $0.74(0.09-5.79)$ & 1.00 \\
Molecular subtypes & 154 & $1.17(0.51-2.69)$ & 0.51 \\
HR-/HER2+ vs. HR+/HER2- & & $1.87(0.63-5.54)$ & 0.77 \\
HR+/HER2+ vs. HR+/HER2- & & $1.52(0.21-11.3)$ \\
TN vs. HR+/HER2- & 89 & - & 0.48 \\
Ki67, $\geq 20$ vs. <20\% & 160 & $0.28(0.10-0.84)$ \\
TNM, N1-3 vs. N0 & - & $0.23(0.05-1.03)$ \\
TNM, M1 vs. M0x & 110 & 0.89 \\
Clinical response, yes vs. no & 89 & 0.26 \\
pCR, yes vs. no & & 0.681 \\
\hline
\end{tabular}

Concerned 160 non-metastatic (M0) patients at diagnosis. HR, hormonal receptor; HER2, human epidermal growth factor receptor 2; TN, triple negative; N, nodes; $\mathrm{M}$, metastasis; pCR, pathological complete response; HR, hazard ratio; CI, confidence interval.

in previous studies $(18,19)$ where high HER2 positive and TN frequencies ( $>30$ and $>20 \%$, respectively) confer a bad prognosis to IBC, despite a higher rate of clinical and histological response to chemo and targeted therapy. IBC aggressiveness is also confirmed in our series, by an increased average Ki-67 expression (>20\%), a nuclear non-histone protein present at low levels in quiescent cells but increased in proliferating cells (20,21). Masuda et al (22) showed that there was no significant difference in clinical outcomes in IBC between ER/PR-positive/HER2-positive, ER/PR-positive/HER2-negative, and ER/PR-negative/HER2positive subtypes. In our series, TN subtype was associated with a high pathological grade (III) comparatively to the other subtypes. In a retrospective study of 240 patients, treated at the MD Anderson Cancer Center in six prospective trials between 1973 and 2000, a 25\% pCR rate was observed after FEC-paclitaxel vs. $10 \%$ after FEC alone $(23,24)$. In the same study of Cristofanilli et al (24), pCR was shown to be a strong predictor for prognosis and a better DFS tended to be associated with the presence of $\mathrm{pCR}\left(\mathrm{P}=6 \times 10^{-2}\right)$. The same was concluded in our study with $35 \%$ of $\mathrm{pCR}$ in patients receiving paclitaxel plus anthracycline-containing regimen and was found associated with a better MFS $\left(\mathrm{P}=3 \times 10^{-2}\right)$.

Histologic grades and involvement of axillary nodes are, like in 'classical BC', others important prognostic factors, for the risk of recurrence and/or overall and disease-free survival $(25,26)$.

Multimodality therapy with systemic neoadjuvant chemotherapy and/or targeted therapy, followed by loco-regional surgery and radiotherapy, became the standard approach and improved IBC survival (27). Following previous studies, we also observed that for IBC management in Tunisia, the introduction of taxanes and trastuzumab in the neoadjuvant setting improves the prognosis of IBC. The introduction of taxanes showed benefit in metastatic breast cancer $(11,22)$ and the administration of biological therapy with the monoclonal antibody trastuzumab was recommended for a patient with HER2-positive disease (22). While anthracycline- and taxane-containing regimens are most commonly prescribed, the optimal chemotherapy regimen and sequence of agents have yet to be defined (28). Multimodality treatment is the standard treatment for IBC, and induction of chemotherapy followed by surgery and radiotherapy seems to be the best sequence.

To our knowledge, our study is the first to examine IBC by clinicopathological characteristics and by subtypes based on hormonal and HER2 status in a large series of patients from one institution in a short period of 5 years with the same protocol treatment notably the introduction of target therapy. The limits of our study were the retrospective nature and associated biases such as missing data with $20 \%$ of patients lost for follow-up and the insufficiency of the number of patients per subtype for analysis, resulting in limited statistical power.

In conclusion, our study confirms, the younger age of Tunisian IBC patients, compared to US and European series, the poor prognosis histo and immunohistochemical features with the predominance of HR-, HER2+ and TN subtypes. These characteristics could explain the poor OS and MFS observed lower than 50 and $40 \%$, respectively, despite the multidisciplinary approach used for combined medical and 
loco-regional treatment. Identify new therapeutic targets to treat IBCs more efficiently constitutes a priority and a real hope for a patient.

\section{Acknowledgements}

The authors would like to thank the teams at the Department of Medical Oncology, University Hospital Abderrahmane Mami, Salah Azaiez Institute of Tunis, Inserm and Paoli-Calmettes Institute. The authors would also like to thank the Ministère d'Enseignement Supérieur de la Recherche Scientifique de Tunisie and Université de Tunis El Manar.

\section{Funding}

The present study was supported by The Joint Committee of University Cooperation PHC Utique in a Franco-Tunisian mixed research project (received by MaM; grant no. 15G0805), The National League Against Cancer (received by DB; grant no. R16009AA), SIRIC (grant no. INCa-DGOS-Inserm 6038) and the Pink Ribbon (grant no. R16024AA).

\section{Availability of data and materials}

The datasets used and/or analyzed during the current study are available from the corresponding author on reasonable request.

\section{Authors' contributions}

MaM conceived the project. MaM, NM, KR, AG and SA contributed by providing the data, and conceived and designed experiments. MaM, PF and FB analyzed the data. MaM and $\mathrm{HB}$ wrote the manuscript. HB, NM, DB, MC, AG and MoM revised the manuscript critically for important intellectual content, and MC, DB and MoM gave final approval of the version to be published.

\section{Ethics approval and consent to participate}

The present study was approved by the Institutional Ethics Committee of Institut Salah Azaiez (approval no. 1646).

\section{Patient consent for publication}

Patients gave their informed consent for being included in the hospital breast cancer database for research purpose and publication.

\section{Competing interests}

The authors declare that they have no competing interests.

\section{References}

1. Boussen H, Bouzaiene H, Ben Hassouna J, Gamoudi A, Benna $\mathrm{F}$ and Rahal K: Inflammatory breast cancer in Tunisia: Reassessment of incidence and clinicopathological features. Semin Oncol 35: 17-24, 2008.

2. Labidi SI, Mrad K, Mezlini A, Ouarda MA, Combes JD, Ben Abdallah M, Ben Romdhane K, Viens P and Ben Ayed F: Inflammatory breast cancer in Tunisia in the era of multimodality therapy. Ann Oncol 19: 473-480, 2008.
3. Dawood S, Merajver SD, Viens P, Vermeulen PB, Swain SM, Buchholz TA, Dirix LY, Levine PH, Lucci A, Krishnamurthy $\mathrm{S}$, et al: International expert panel on inflammatory breast cancer: Consensus statement for standardized diagnosis and treatment. Ann Oncol 22: 515-523, 2011.

4. Fouad TM, Barrera AMG, Reuben JM, Lucci A, Woodward WA, Stauder MC, Lim B, DeSnyder SM, Arun B, Gildy B, et al: Inflammatory breast cancer: A proposed conceptual shift in the UICC-AJCC TNM staging system. Lancet Oncol 18: e228-e232, 2017.

5. Li J, Xia Y, Wu Q, Zhu S, Chen C, Yang W, Wei W and Sun S: Outcomes of patients with inflammatory breast cancer by hormone receptor- and HER2-defined molecular subtypes: A population-based study from the SEER program. Oncotarget 8: 49370-49379, 2017.

6. Mirecka J, Korabiowska M and Schauer A: Correlation between the occurrence of Ki-67 antigen and clinical parameters in human breast carcinoma. Folia Histochem Cytobiol 31: 83-86, 1993.

7. Boussen H, Bouzaiene H, Ben Hassouna J, Dhiab T, Khomsi F, Benna F, Gamoudi A, Mourali N, Hechiche M, Rahal K and Levine PH: Inflammatory breast cancer in Tunisia: Epidemiological and clinical trends. Cancer 116 (Suppl 11): 2730-2735, 2010

8. Mourali N, Muenz LR, Tabbane F, Belhassen S, Bahi J and Levine PH: Epidemiologic features of rapidly progressing breast cancer in Tunisia. Cancer 46: 2741-2746, 1980.

9. Maalej M, Hentati D, Messai T, Kochbati L, El May A, Mrad K, Romdhane KB, Ben Abdallah M and Zouari B: Breast cancer in Tunisia in 2004: A comparative clinical and epidemiological study. Bull Cancer 95: E5-E9, 2008.

10. Chaher N, Arias-Pulido H, Terki N, Qualls C, Bouzid K, Verschraegen C, Wallace AM and Royce M: Molecular and epidemiological characteristics of inflammatory breast cancer in Algerian patients. Breast Cancer Res Treat 131: 437-444, 2012.

11. Mejri N, Boussen H, Labidi S, Bouzaiene H, Afrit M, Benna F and Rahal K: Inflammatory breast cancer in Tunisia from 2005 to 2010: Epidemiologic and anatomoclinical transitions from published data. Asian Pac J Cancer Prev 16: 1277-1280, 2015.

12. Hirko KA, Soliman AS, Banerjee M, Ruterbusch J, Harford JB, Chamberlain RM, Graff JJ, Merajver SD and Schwartz K: Characterizing inflammatory breast cancer among Arab Americans in the California, Detroit and New Jersey Surveillance, Epidemiology and End Results (SEER) registries (1988-2008). Springerplus 2: 3, 2013.

13. Goldner B, Behrendt CE, Schoellhammer HF, Lee B and Chen SL: Incidence of inflammatory breast cancer in women, 1992-2009, United States. Ann Surg Oncol 21: 1267-1270, 2014.

14. Levine PH and Veneroso C: The epidemiology of inflammatory breast cancer. Semin Oncol 35: 11-16, 2008.

15. Charafe-Jauffret E, Mrad K, Intidhar Labidi S, Ben Hamida A, Ben Romdhane K, Ben Abdallah M, Ginestier C, Esterni B, Birnbaum D, Ben Ayed F and Xerri L: Inflammatory breast cancers in Tunisia and France show similar immunophenotypes. Breast 16: 352-358, 2007.

16. Veneroso C, Siegel R and Levine PH: Early age at first childbirth associated with advanced tumor grade in breast cancer. Cancer Detect Prev 32: 215-223, 2008.

17. Schairer C, Li Y, Frawley P, Graubard BI, Wellman RD, Buist DS, Kerlikowske K, Onega TL, Anderson WF and Miglioretti DL: Risk factors for inflammatory breast cancer and other invasive breast cancers. J Natl Cancer Inst 105: 1373-1384, 2013.

18. Obr AE and Edwards DP: The biology of progesterone receptor in the normal mammary gland and in breast cancer. Mol Cell Endocrinol 357: 4-17, 2012.

19. Zwart W, Theodorou V and Carroll JS: Estrogen receptor-positive breast cancer: A multidisciplinary challenge. Wiley Interdiscip Rev Syst Biol Med 3: 216-230, 2011.

20. Stuart-Harris R, Caldas C, Pinder SE and Pharoah P: Proliferation markers and survival in early breast cancer: A systematic review and meta-analysis of 85 studies in 32,825 patients. Breast 17: 323-334, 2008.

21. Viale G, Giobbie-Hurder A, Regan MM, Coates AS, Mastropasqua MG, Dell'Orto P, Maiorano E, MacGrogan G, Braye SG, Ohlschlegel C, et al: Prognostic and predictive value of centrally reviewed Ki-67 labeling index in postmenopausal women with endocrine-responsive breast cancer: Results from Breast International Group Trial 1-98 comparing adjuvant tamoxifen with letrozole. J Clin Oncol 26: 5569-5575, 2008. 
22. Masuda H, Brewer TM, Liu DD, Iwamoto T, Shen Y, Hsu L, Willey JS, Gonzalez-Angulo AM, Chavez-MacGregor M, Fouad TM, et al: Long-term treatment efficacy in primary inflammatory breast cancer by hormonal receptor- and HER2-defined subtypes. Ann Oncol 25: 384-391, 2014

23. Cristofanilli M, Gonzalez-Angulo AM, Buzdar AU, Kau S-W, Frye DK and Hortobagyi GN: Paclitaxel improves the prognosis in estrogen receptor negative inflammatory breast cancer: The M. D. Anderson Cancer Center experience. Clin Breast Cancer 4: 415-419, 2004

24. Cristofanilli M, Buzdar AU, Sneige N, Smith T, Wasaff B, Ibrahim N, Booser D, Rivera E, Murray JL, Valero V, et al: Paclitaxel in the multimodality treatment for inflammatory breast carcinoma. Cancer 92: 1775-1782, 2001.

25. Elston CW and Ellis IO: Pathological prognostic factors in breast cancer. I. The value of histological grade in breast cancer: Experience from a large study with long-term follow-up. Histopathology 19: 403-410, 1991.

26. Jatoi I, Hilsenbeck SG, Clark GM and Osborne CK: Significance of axillary lymph node metastasis in primary breast cancer. J Clin Oncol 17: 2334-2340, 1999.
27. Pisansky TM, Schaid DJ, Loprinzi CL, Donohue JH, Schray MF and Schomberg PJ: Inflammatory breast cancer: Integration of irradiation, surgery and chemotherapy. Am J Clin Oncol 15: 376-387, 1992.

28. Cristofanilli M, Valero V, Buzdar AU, Kau SW, Broglio KR, Gonzalez-Angulo AM, Sneige N, Islam R, Ueno NT, Buchholz TA, et al: Inflammatory breast cancer (IBC) and patterns of recurrence: Understanding the biology of a unique disease. Cancer 110: 1436-1444, 2007. Attribution-NonCommercial-NoDerivatives 4.0 International (CC BY-NC-ND 4.0) License. 